\title{
Ionospheric effects on the $F$ region during the sunrise for the annular solar eclipse over Taiwan on 21 May 2012
}

\author{
Y. J. Chuo \\ Department of Information Technology, Ling Tung University, 408 Taichung, ROC, Taiwan
}

Correspondence to: Y. J. Chuo (yjchuo@teamail.ltu.edu.tw)

Received: 8 June 2013 - Revised: 2 October 2013 - Accepted: 4 October 2013 - Published: 1 November 2013

\begin{abstract}
On 21 May 2012 (20:56, Universal Time; UT, on 20 May), an annular solar eclipse occurred, beginning at sunrise over southeast China and moving through Japan, sweeping across the northern Pacific Ocean, and completing its passage over the western United States at sunset on 20 May 2012 (02:49 UT, 21 May). We investigated the eclipse area in Taiwan, using an ionosonde and global positioning system (GPS) satellite measurements. The measurements of $f o \mathrm{~F}_{2}, h m \mathrm{~F}_{2}$, bottomside scale height around the peak height $\left(H_{m}\right)$, and slab thickness $\left(B_{0}\right)$ were collected at the ionosonde station at Chung-Li Observatory. In addition, we calculated the total electron content (TEC) to study the differences inside and outside the eclipse area, using 3 receivers located at Marzhu (denoted as MATZ), Hsinchu (TNML), and Henchun (HENC). The results showed that the $f o \mathrm{~F}_{2}$ values gradually decreased when the annularity began and reached a minimum level of approximately $2.0 \mathrm{MHz}$ at 06:30 LT. The $h m \mathrm{~F}_{2}$ immediately decreased and then increased during the annular eclipse period. The TEC variations also appeared to deplete in the path of the eclipse and opposite the outside passing area. Further, the rate of change of the TEC values ( $\mathrm{dTEC} / \mathrm{d} t$ measured for $15 \mathrm{~min}$ ) was examined to study the wave-like fluctuations. The scale height near the $\mathrm{F}_{2}$ layer peak height $\left(H_{m}\right)$ also decreased and then increased during the eclipse period. To address the effects of the annular eclipse in the topside and bottomside ionosphere, this study provides a discussion of the variations between the topside and bottomside ionospheric parameters during the eclipse period.
\end{abstract}

Keywords. Ionosphere (Ionospheric disturbances)

\section{Introduction}

Solar eclipses are natural phenomena involving the Sun, the Moon, and their relationship to the Earth. This phenomenon provides valuable information on topside and bottomside ionospheric processes, particularly when the event occurs during sunrise. The ionosphere can be divided into two parts according to the $\mathrm{F}_{2}$ layer peak; altitudes higher than the $\mathrm{F}_{2}$ peak are considered the topside ionosphere, and altitudes lower than the $\mathrm{F}_{2}$ peak are considered the bottomside ionosphere. The physical processes differ in the topside and bottomside ionospheres. The topside ionosphere is dominated by diffusion mechanisms and the bottomside is controlled by photochemical mechanisms. Recent ionospheric studies have used observations and model simulations, indicating that the ionospheric eclipse effect is a major event in the lower altitude ionosphere ( $\mathrm{E}$ and $\mathrm{F}_{1}$ layers) because these layers are associated with photochemical ionization. By contrast, the $\mathrm{F}_{2}$ layer remains ambiguous, showing various responses in various areas. Nayak et al. (2012) studied the ionospheric response of the 15 January 2010 eclipse over an equatorial observation station, indicating that the electron density in the $\mathrm{F}_{2}$ region decreased slightly, beginning at lower heights and extending to the layer peak at approximately noon. In addition, Jakowski et al. (2008) studied the ionospheric behavior of the annular eclipse that occurred before noon on 3 October 2005 over Europe. They observed a wave-like structure moving through the ionospheric $\mathrm{F}$ layer region. Those results revealed an eclipse-induced $f o F_{2}$ depression in the ionosphere with a time delay from the maximum obscuration. Furthermore, Cheng et al. (1992) studied the ionospheric effects of the solar eclipse on 23 September 1987 around the equatorial anomaly crest region during the post-sunrise period, finding a decrease in the $\mathrm{E}$ layer and $f_{o} \mathrm{~F}_{1}$ but an increase in $f o \mathrm{~F}_{2}$. 
This indicates that the ionospheric response to solar eclipses varies depending on the place and the time of the occurrence, particularly in the $\mathrm{F}_{2}$ region. The eclipse effect was larger at midday than in the morning and afternoon (Le et al., 2008, 2009; Nayak et al., 2012), and high solar activity was more obvious than low solar activity (Nayak et al., 2012). In the present study, the event onset time was during the sunrise, and the $F_{1}$ and $\mathrm{E}$ layers were not formed during that period. Therefore, the ionospheric eclipse effect is mainly associated with the $\mathrm{F}_{2}$ layer and topside ionosphere.

We used two data sets from the ionosonde and global positioning system (GPS) for the annular solar eclipse over Taiwan on 21 May 2012, which was located near the crest of the equatorial ionization anomaly (EIA) area. On 20 May 2012, an annular solar eclipse began, and was observed in the early morning at 20:56 UT (04:56 LT on 21 May 2012), providing a unique opportunity to study the formation of the daytime ionosphere and the variations in the topside and bottomside ionosphere. During the eclipse, the geomagnetic status was quiet at the solar maximum. The present study shows the ionospheric response during the maximum phase of the eclipse, lasting several hours. This elucidates the dynamic response of the ionosphere to the eclipse-induced traveling ionospheric disturbances.

\section{Data and analysis methods}

Ionosonde and GPS data sets were used to examine the variations in $f o \mathrm{~F}_{2}, h m \mathrm{~F}_{2}, B_{0}, H_{m}$, total electron content (TEC), and equivalent slab thickness $(\tau)$ during the eclipse. Figure 1 shows the locations of the Chung-Li ionosonde $\left(25.0^{\circ} \mathrm{N}, 121.1^{\circ} \mathrm{E}\right)$, indicated by the red circle and the GPS receivers at Marzhu $\left(26.17^{\circ} \mathrm{N}, 119.92^{\circ} \mathrm{E}\right.$; marker name as MATZ), Hsinchu $\left(24.80^{\circ} \mathrm{N}, 120.99^{\circ} \mathrm{E}\right.$; TNML), and Henchun $\left(22.0^{\circ} \mathrm{N}, 120.75^{\circ} \mathrm{E}\right.$; HENC), marked on the map with blue squares. The annular path of the eclipse is represented by the black dashed lines and the central line indicates the annular path width and the maximum annularity. The ionosonde measurements were routinely recorded every $15 \mathrm{~min}$. The ionosonde data sets for $f o \mathrm{~F}_{2}, h m \mathrm{~F}_{2}, H_{m}$, and $B_{0}$ were used. The bottomside iTEC was calculated from manually scaled ionograms in the true-height inversion program, POLAN (Titheridge, 1985). The program calculates the ionospheric electron density true-height profiles and measurements for $f o \mathrm{~F}_{2}, h m \mathrm{~F}_{2}$, and $H_{m}$. The $B_{0}$ values were derived using Gulyaeva's method (Gulyaeva, 1987, 2007), which provides a model for the $h m \mathrm{~F}_{2}$ and the altitude of half-electron density of the $F_{2}$ peak height. The topside electron density profile was reconstructed using the $\alpha$-Chapman function, according to the method proposed by Reinisch and Huang (2001). To reconstruct the Ne profile above the $\mathrm{F}_{2}$ layer peak height, they assumed the fixed scale height $H_{m}$ in the topside ionosphere. To investigate the effect of the annular solar eclipse on the ionospheric parameters, the eclipseday observations were compared with a monthly mean as the

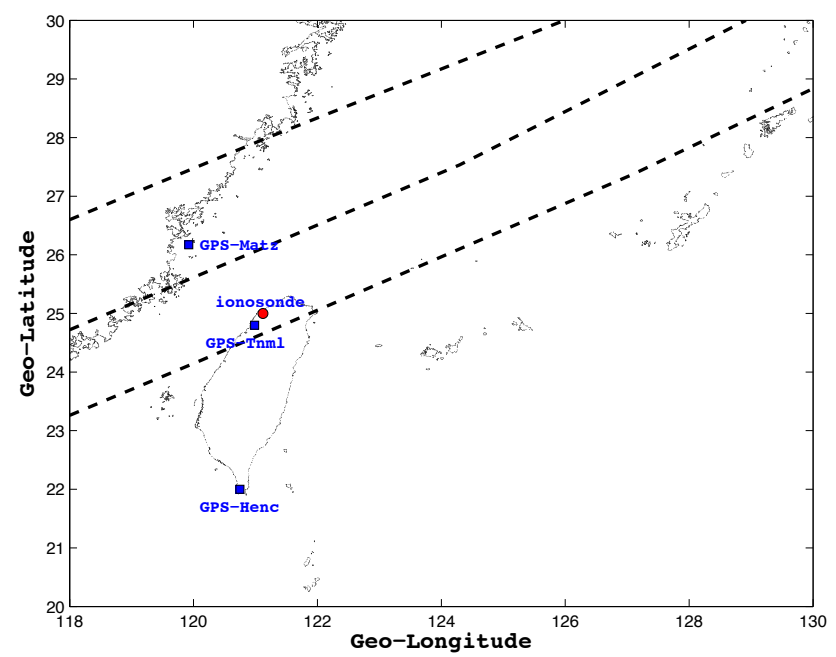

Fig. 1. Map showing the path of the solar eclipse on 21 May 2012 (dashed lines) and the location of observations over Taiwan. The ionosonde and GPS stations are shown in a filled red circle and blue squares, respectively.

control day. Although the ionosonde can record bottomside ionospheric data, it only detects a single point. Three GPS ground receivers were therefore used to calculate the vertical TEC for studying the ionospheric response. The MATZ and TNML receivers were located along the path of the eclipse, and the HENC receiver was outside of the path. In this study, the GPS TEC techniques were integrated with data from several area surveys to analyze the significance of the results and their correlation with the ionosonde sounding features. The vertical TEC data was derived from the GPS signals by using the carrier phase of the dual-frequency method (Liu et al., 1996). The ionospheric equivalent slab thickness $(\tau)$ was derived from the ratio of TEC to $N m \mathrm{~F}_{2}$, where $N m \mathrm{~F}_{2}$ is the peak electron density at the peak height. This study analyzed the effect of eclipse-induced changes in the topside and bottomside $F_{2}$ layer regions of the ionosphere were analyzed.

\section{Results and discussion}

The eclipse occurred during a period of quiet solar activity in which the Dst index varied from -10 to -13 (nT). The event provided the opportunity to study the ionospheric responses to the eclipse. Figure 2 shows the varying ionospheric parameters of $f o \mathrm{~F}_{2}, h m \mathrm{~F}_{2}, H_{m}$, and $B_{0}$ values from 18 to 23 May 2012. The dotted lines represent the daily variation and the solid lines with two red lines denote the monthly mean with error bar values of the previously mentioned ionospheric parameters. The three vertical dashed lines represent the first contact time (05:07 LT), second contact time (06:10 LT), and the end time (07:22 LT) for the annular eclipse over Taiwan. Figure 2a shows an obvious and rapid decrease in $f o \mathrm{~F}_{2}$ at the second contact time (06:10 LT) 

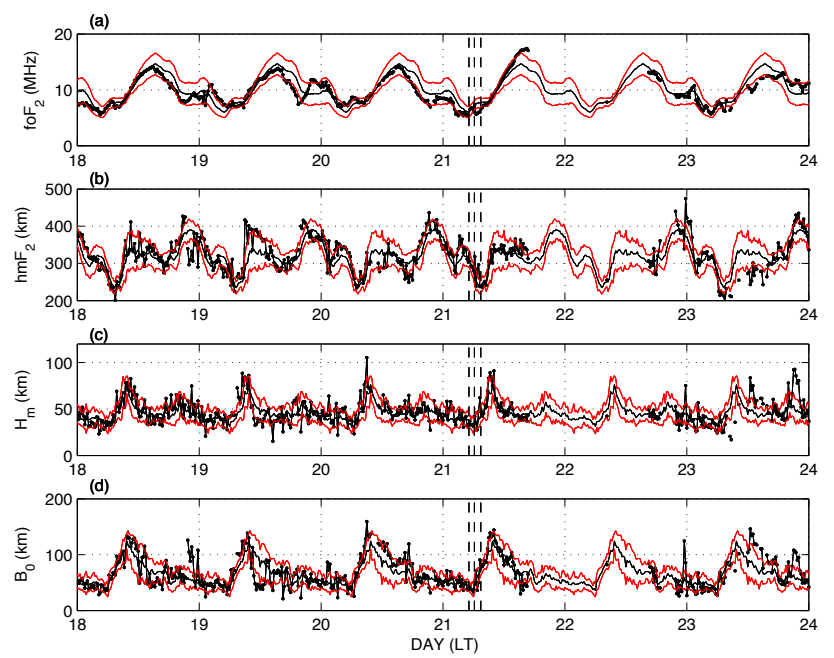

Fig. 2. Variations in (a) $f o \mathrm{~F}_{2}$, (b) $h m \mathrm{~F}_{2}$, (c) $H_{m}$, and (d) $B_{0}$ parameters from 18 to 23 May 2012. The dotted, solid, and red solid lines denote the observed, monthly mean, and error bar values, respectively. The vertical dashed lines show the onset, second contact, and end time of the annular solar eclipse over Taiwan.

on 21 May 2012. Although a similar drop in $f o \mathrm{~F}_{2}$ around the sunrise period was observed on days 18 and 23, the decrease was slight and gradual and also within the control curve. However, this substantial and sudden decrease in the $f o \mathrm{~F}_{2}$ was lower than the control curve during the maximum to end phase of the eclipse on 21 May and was not observed at the same period on the other days. The second contact time was also the maximum obscuration of the annular eclipse over Taiwan. The maximum decrease in the $f o \mathrm{~F}_{2}$ occurred at 06:30 LT, with an approximate value of $2.0 \mathrm{MHz}$. In addition, an increase in the $f o \mathrm{~F}_{2}$ was observed after the end of the annular eclipse at noon. The maximum increase was approximately 3.6 MHz. Regarding the lack of data from 16:45 LT on Day 21 to 07:45 LT on Day 22, no ionograms were produced by the ionosonde. A rapid fluctuation was observed in the $h m \mathrm{~F}_{2}$ compared with the control values between 06:00 LT and 07:00 LT (Fig. 2b). The $H_{m}$ and B0 results also showed a fluctuation during the annular eclipse (Fig. $2 \mathrm{c}$ and d). A sudden increase was observed both in $H_{m}$ and $B_{0}$ after the end of the annular eclipse between 08:30 LT and 11:15 LT. Figure 3 shows the variations in $f o \mathrm{~F}_{2}, h m \mathrm{~F}_{2}, H_{m}$, and $B_{0}$ between 03:00 LT and 09:00 LT on 21 May 2012. The six lines denote the observed values (black dotted lines), monthly mean values (red lines), and quiet geomagnetic day values (green lines, blue lines, dashed lines, and solid lines indicate the values on 18, 19, 20, and 23 May 2012, respectively). Figure 3 shows the decrease in the $f o \mathrm{~F}_{2}$ beginning at 06:00 LT and reaching the minimum value $(5.63 \mathrm{MHz})$ at $06: 15 \mathrm{LT}$, then increasing gradually until 07:30 LT and returning to the typical normal day value. In general, the electron density and the $f o \mathrm{~F}_{2}$ values increased gradually after sunrise
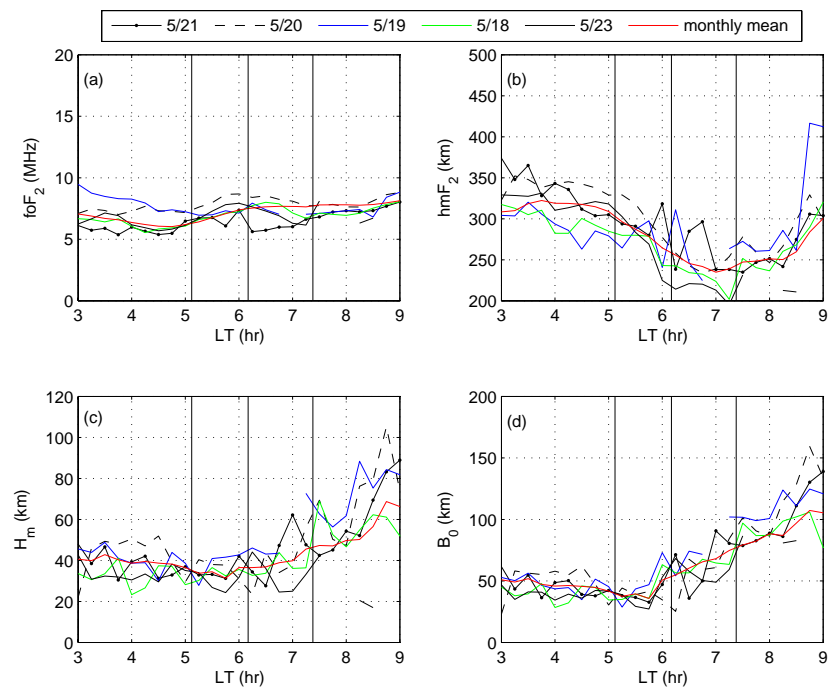

Fig. 3. The detailed variations in (a) $f o \mathrm{~F}_{2}$, (b) $h m \mathrm{~F}_{2}$, (c) $H_{m}$, and (d) $B_{0}$ parameters from 03:00 LT to 09:00 LT on 21 May 2012. The dotted, green, blue, dashed, solid, and red solid lines show the variation on 21, 18, 19, 20, and 23 May and the monthly mean, respectively. The vertical solid lines represent the onset, second contact, and ending time of the annular solar eclipse.

(approximately 06:00 LT); however, the $f o \mathrm{~F}_{2}$ decreased between 06:00 LT and 07:15 LT on 21 May 2012 (Fig. 3a). The maximum obscuration occurred at 06:10 LT and the eclipse ended at 07:23 LT. We suggest that the $f o \mathrm{~F}_{2}$ followed the variation in local solar radiation during the eclipse. This result contrasts with the study by Cheng et al. (1992) of the annular eclipse of 23 September 1987 over the EIA crest region, which showed an increase in the $f o \mathrm{~F}_{2}$ during the eclipse period and the mainly effect controls in the $f o \mathrm{~F}_{1}$ values. Moreover, Cheng et al. (1992) showed that a time delay in the $f o \mathrm{~F}_{2}$ decreases after the maximum solar obscuration. In this case, the eclipse occurred approximately during the sunrise period, which is when the $f o \mathrm{~F}_{2}$ can be eclipse-induced and possibly lost to recombination and production by photoionization. With the increase in eclipse obscuration, the equation for electron equilibrium in the ionosphere can be written as follows:

$\mathrm{d} N / \mathrm{d} t=q(t)-\alpha N^{2}$

and

$\mathrm{d} N / \mathrm{d} t=q(t)-\beta N^{2}$

where $\alpha$ and $\beta$ are the recombination coefficients, $q(t)$ is the rate of electron production, and $N$ is the electron density (Davies, 1990). According to Cheng et al. (1992) the variation in the $F_{1}$ layer peak electron density during the solar eclipse period can be expressed as the quadratic and linear function of solar radiation as follows: 
$S=\left[(N m \mathrm{~F} 1)_{e} /(N m \mathrm{~F} 1)_{c}\right]^{2}=\left[(f o \mathrm{~F} 1)_{e} /(f o \mathrm{~F} 1)_{c}\right]^{4}$

and

$S=\left[(N m \mathrm{~F} 1)_{e} /(N m \mathrm{~F} 1)_{c}\right]=\left[(f o \mathrm{~F} 1)_{e} /(f o \mathrm{~F} 1)_{c}\right]^{2}$,

where $S$ is the fraction of uneclipsed solar disk, and the subscripts $e$ and $c$ denote the eclipse and control days, respectively. In this study, we replaced the $F_{1}$ layer with the $F_{2}$ layer peak electron density to examine whether the $F_{2}$ layer is dominated by diffusion mechanisms.

Figure 4 shows the varying fractions of obscuration; the red solid circles and crosses represent the variation in the $f o \mathrm{~F}_{2}$ to the second power, and the large hollow circles and asterisks represent the variation in the $f o \mathrm{~F}_{2}$ to the fourth power on 21 and 20 May, respectively. The results show that the variation in fourth-power $f o \mathrm{~F}_{2}$ follows the variation in solar obscuration during the annular eclipse more closely than does the second-power $f o \mathrm{~F}_{2}$ on 21 May. In other words, the decrease in electron density is dominated by ionic recombination rather than attachment. Furthermore, the solar radiation increased until the local noon on 21 May. At the same time, the solar radiation decreased to close to the zero line on 20 May. In other words, the solar radiation and transport process in the $\mathrm{F}$ region were delayed and lasted until the local noon after the annular eclipse. We also studied the shape variations in the $\mathrm{F}_{2}$ layer. In general, the value of $h m \mathrm{~F}_{2}$ should decrease by degrees during the dawn period. Figure $3 \mathrm{~b}$ shows an abrupt variation in the $h m \mathrm{~F}_{2}$ from $279 \mathrm{~km}$ to $318 \mathrm{~km}$ that fluctuated again and returned to typical values at 07:00 LT. This suggests that the ionosphere produced a wave-like fluctuation after the first contact with the eclipse because of the $f_{o} \mathrm{~F}_{2}$ with the same phase from 05:45 to $06: 15 \mathrm{LT}$. The $h m \mathrm{~F}_{2}$ then increased from $238 \mathrm{~km}$ to $296 \mathrm{~km}$ from 06:15 to 06:45 LT. This is associated with the maximum obscuration of the eclipse and the redistribution, suggesting that the plasma modifies the shape of the electron density. Jakowski et al. (2008) observed that the substantial depletion of ionization could suggest an enhancement in the $h m \mathrm{~F}_{2}$. Le et al. (2009) performed a modeling study, indicating that a considerable amount of enhancement should be expected in the $h m \mathrm{~F}_{2}$ on the eclipse day, compared with the control days. The present study supports these suggestions and results. Le et al. (2009) also indicated that the uplift in the $h m \mathrm{~F}_{2}$ was driven by the vertical drift. Furthermore, we examined the ionospheric scale height around the peak height $\left(H_{m}\right)$, which was considered to be associated with the sunrise during the daytime (Lee and Reinisch, 2007). The result showed a small fluctuation between 06:00 LT and 07:00 LT and an apparent increase at 07:00 LT (Fig. 3c). The result agrees with the obscuration of the eclipse, with a $15 \mathrm{~min}$ time delay, and the lowest value occurring at 06:30 LT. In addition, we examined the $B_{0}$ values, observing oscillation between 06:00 LT and 07:00 LT and a negative correlation with the $h m \mathrm{~F}_{2}$ (Fig. 3d). The variation in $B_{0}$ increased from 05:45 to $06: 15 \mathrm{LT}$ and

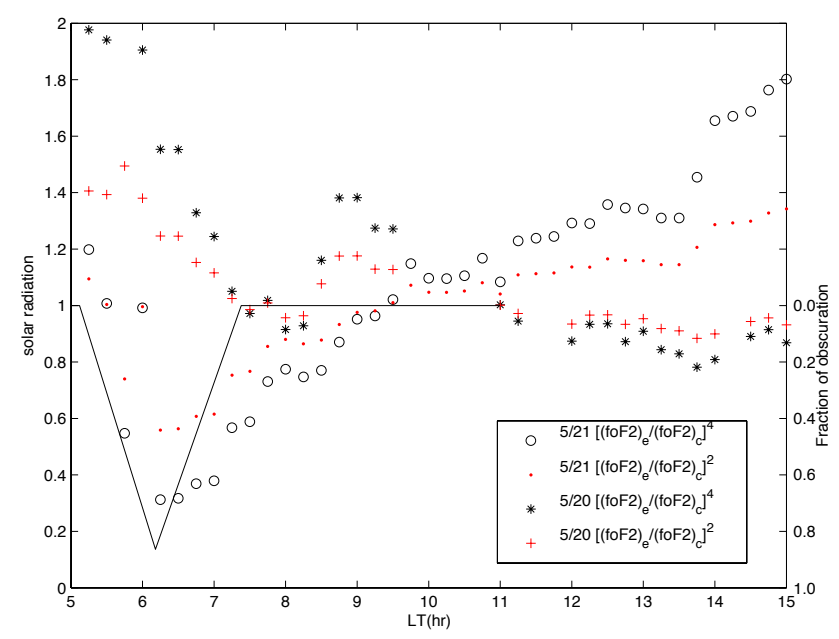

Fig. 4. Variations in the fraction of obscuration (solid line), the second power (red dots and crosses), and the fourth power (circles and asterisk) of $f o \mathrm{~F}_{2}$ on 21 and 20 May 2012, respectively.

abruptly decreased at 06:30 LT. Figures 2 and 3 show that the ionospheric bottomside $\mathrm{F}_{2}$ layer reduced the electron density and also drove the fluctuation in the ionosphere during the annular eclipse. Furthermore, the results indicate that the bottomside ionospheric eclipse response was associated with the change in ionospheric shape that was driven by the magnetic equatorial $\boldsymbol{E} \times \boldsymbol{B}$ vertical drift and redistribution.

We used GPS data to examine variations in TEC and the topside ionosphere. Figure 5 shows the variation in TEC measurements from the MATZ, TNML, and HENC receivers. The red and solid lines represent the observed and control day (monthly mean) values, respectively. The two dashed lines denote the range of mean with error bar values. The results show that TEC decreased during the annular eclipse, according to the MATZ and TNML receivers, but not the HENC receiver. Figure 5 shows that the depletion in electron content between 06:00 LT and 07:00 LT was not observed by the HENC receiver. The annular eclipse depleted electron content; however, this effect occurred only in the path of the eclipse. Additionally, the results show an apparent increase in the maximum deviation value of approximately $20 \mathrm{TECu}\left(1 \mathrm{TECu}=10^{16} \mathrm{el} \mathrm{m}^{-2}\right)$ to $30 \mathrm{TECu}$ between 15:00 LT and 21:00 LT on 21 May 2012 for all receivers. This increase in electron content is observed in the $f o F_{2}$ (Fig. 2a), caused by both the solar radiation and the transport processes. Figure 4 shows an increase in solar radiation that lasted until the local noon on 21 May. Furthermore, at sunrise, the drift of ionization was upward and increased with time along with the $N m \mathrm{~F}_{2}$ and the $h m \mathrm{~F}_{2}$ until the time of occurrence of the morning maximum in the $h m \mathrm{~F}_{2}$ (Adeniyi et al., 2007). Furthermore, an apparent increase in TEC during pre-sunrise was observed at MATZ on 21 May. However, the result shows the TEC also decreased at the onset of the eclipse on 21 May. Because of the mechanism of the 

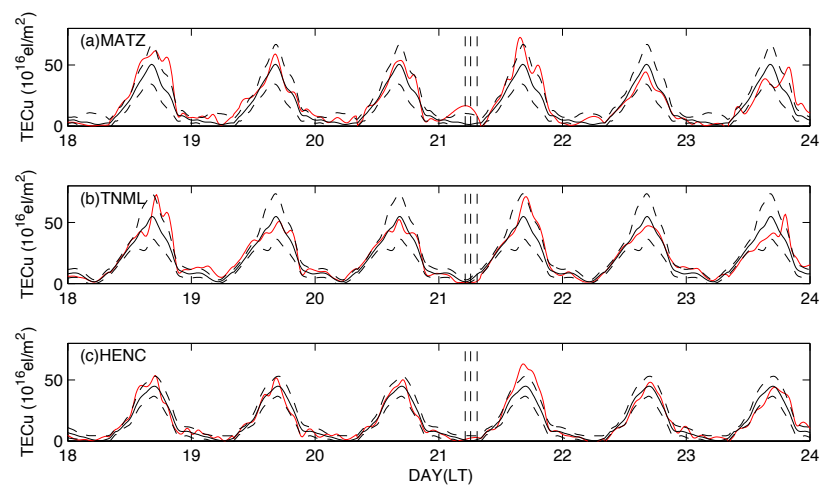

Fig. 5. The variation in GPS TEC observations at stations (a) MATZ, (b) TNML, and (c) HENC from 18 to 23 May 2012. The red, solid, and dashed lines denote the observed, monthly mean, and error bar values, respectively. The vertical dashed lines show the onset, second contact, and ending time of the annular solar eclipse over Taiwan.

apparent increase in TEC during the pre-sunrise on 21 May at MATZ, it remains to be not understood. The GPS TEC data include both the topside and bottomside ionospheres. Therefore, we studied the annular eclipse effect in both the topside and bottomside ionospheres. Figure 6 shows variations in the topside and bottomside iTEC, GPS TEC (from the TNML receiver), and ionospheric equivalent slab thickness $(\tau)$ from 18 to 23 May 2012. The red and solid lines denote the observed and monthly mean values, respectively. The results show a small decrease in the iTEC of the topside and bottomside ionospheres between 06:00 LT and 07:00 LT on 21 May 2012. The reduction was smaller in the topside ionosphere (approximately $2.3 \mathrm{TECu}$ ) than in the bottomside ionosphere (approximately $3 \mathrm{TECu}$ ), but both values were less than the GPS TEC (approximately 7 TECu). An increase occurred in the bottomside iTEC between 09:00 LT and 13:00 LT and then reduced in the bottomside ionosphere. Concurrently, an increase was observed in the topside iTEC, occurring later than the bottomside iTEC at 12:15 LT. This indicates that the ionization process began in the bottomside ionosphere and that the topside ionosphere was affected by the fountain effect, which increased the topside ionospheric electron content. Figure $2 \mathrm{c}$ and d show an increase in the $H_{m}$ and $B_{0}$ after the annular eclipse. The variation in $B_{0}$ is strongly associated with the zenith angle of the sun and the $H_{m}$ is highly correlated with the $B_{0}$. In other words, the eclipse ends in this area, increasing the ionization in the $F$ region and growth of the $F_{1}$ layer. The diffusion effect results from the equatorial fountain effect, which redistributes the ionization. Figure $6 \mathrm{a}$ and $\mathrm{b}$ show that the increase in the ionospheric electron content in the topside and bottomside ionospheres was greater in the topside ionosphere between 11:00 LT and 13:00 LT. Although the iTEC increased, the TEC was not observed between 11:00 LT and 13:00 LT (Fig. 6c). The ionospheric equivalent slab thickness $(\tau)$ did
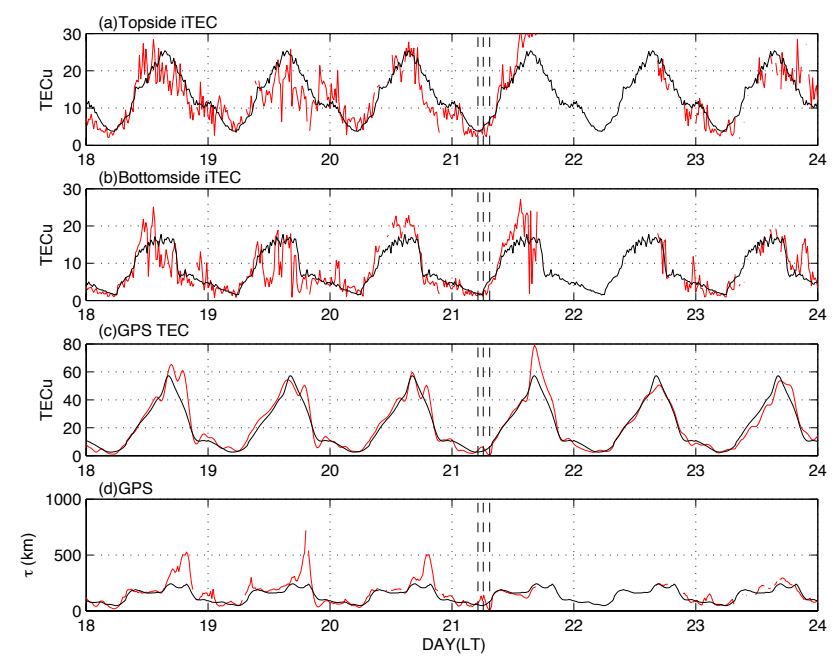

Fig. 6. Variations of (a) topside iTEC, (b) bottomside iTEC, (c) GPS TEC, and (d) ionospheric equivalent slab thickness $(\tau)$ from 18 to 23 May 2012. The red and solid lines denote the observed and monthly mean values, respectively. The vertical dashed lines represent the onset, second contact, and ending time of the annular solar eclipse on 21 May 2012.

not show substantial changes during this period because its value was measured by the ratio of TEC to the $N m \mathrm{~F}_{2}$ and neither value showed substantial changes. The $\tau$ showed an increase at the first contact with the annular eclipse and a decrease after the second contact. A second decrease was observed between 11:30 LT and 14:00 LT (Fig. 6d). Comparing the variations in $B_{0}$ (Fig. 2d) and $\tau$ (Fig. 6d), we determined that $\tau$ decreases more than $B_{0}$ does during the maximum obscuration period of the annular eclipse. This indicates that $\tau$ is more sensitive to solar eclipses than $B_{0}$ is. Additionally, a decrease in $B_{0}, H_{m}$, and $\tau$ was observed between 14:00 LT and 16:30 LT, and was associated with an increasing $h m \mathrm{~F}_{2}$ and an increased bottomside electron density (Figs. 2b and 6). This result was also associated with a time delay in increasing TEC values. Regarding the increase in electron density, the diffusion effect is driven by the equatorial fountain effect. As the eclipse moved north toward Japan and the equatorial fountain effect progressed through Taiwan, the ionization and production were increased by solar radiation, causing an increase in electron densities in Taiwan after the annular eclipse. This suggests that the variations in the $\mathrm{F}_{2}$ layer over Taiwan during an annular eclipse are controlled by both the ionization from solar radiation and the transporting of the equatorial fountain effect.

Another phenomenon, an atmospheric gravity wave (AGW), can be generated by the shadow of the Moon during the eclipse (Chimonas and Hines, 1970; Chimonas, 1970; Cohen, 1984; Cheng et al., 1992; Liu et al., 2011). A bow wave can be produced by the supersonic motion of the shadow of the Moon through the atmosphere along the eclipse path. However, this phenomenon is not observed in 
every eclipse. We therefore determined whether an AGW was present following the 21 May 2012 event. We examined the varying true heights at fixed frequencies of $4.0 \mathrm{MHz}$ to $12.0 \mathrm{MHz}$, step-shifted by $0.5 \mathrm{MHz}$ at $15 \mathrm{~min}$ intervals, using the Chung-Li ionosonde during the annular eclipse (Fig. 7). The four plots represent the reflection altitude values at fixed sounding frequencies from 1 day prior (20 May 2012; Fig. 7a), the occurrence day (21 May 2012; Fig. 7b), the most quiet geomagnetic activity day during the month of the eclipse (27 May 2012; Fig. 7c), and the day after the eclipse (24 May 2012; Fig. 7d). Figure 7a shows a quiet condition from 05:00 LT to 09:30 LT on 20 May 2012. Figure $7 \mathrm{~d}$ also shows a quiet condition except for a fluctuation from 10:00 LT to 11:00 LT and an abrupt increase at 13:30 LT on 24 May 2012. Figure 7b shows a pronounced oscillation over a period of approximately 45 min that was observed in the $\mathrm{F}_{2}$ layer during the eclipse. An apparent oscillation in height was observed from $275 \mathrm{~km}$ to $190 \mathrm{~km}$, which rose to $273 \mathrm{~km}$ between 06:00 LT and 07:00 LT. Therefore, the wave-like oscillation was likely caused by the annular solar eclipse and was possibly a gravity wave. The oscillation observed from $6.0 \mathrm{MHz}$ to the $N m \mathrm{~F}_{2}$ suggests that the gravity wave propagated from a low altitude and moved to a higher altitude. Additionally, subsequent wave-like oscillations developed between 09:00 LT, and 10:00 LT and between 11:30 LT and 15:30 LT on the eclipse day. The fluctuation at 09:00 LT was likely caused by the annular eclipse. This fluctuation was not observed during the same period on the other days. In addition, Fig. 4 shows an increase in the solar radiation from 09:00 LT and lasting quite a while on 21 May 2012. Simultaneously, electron content increased in the bottomside ionosphere (Fig. 6b), but did not exceed that in the topside ionosphere (Fig. 6a). Therefore, the increase in solar radiation led an electron density redistribution to cause a wave-like oscillation at 09:00 LT on 21 May. Due to the fluctuation from 11:30 LT to 15:30 LT, it could have been triggered by other mechanisms and not by the eclipse. Figure $7 \mathrm{c}$ shows a continuous wave-like fluctuation during the daytime on 27 May 2012. Mechanisms of the wave-like fluctuation under quiet geomagnetic activity conditions warrant further research.

We examined the rate of TEC changes $(\mathrm{dTEC} / \mathrm{d} t)$ at 15 min intervals. In Fig. 8 the dashed, dotted, blue, and red solid lines represent the rate of change in TEC for the monthly mean and that for 21 May (the eclipse day), 20 May (the day before the eclipse), and 22 May (the day after the eclipse), at 15 min intervals at all observation stations. TEC decreased during the annular eclipse period at MATZ and TNML, but not at HENC on 21 May. The decrease in the rate of change was not observed during the same period for all three stations on 20 and 22 May, except for a slight decrease on 22 May at MATZ. However, a slight decrease occurred in the rate of change after the end of the eclipse at HENC on 21 May. Moreover, a wave-like structure propagated through the ionosphere between 12:00 LT and 22:00 LT
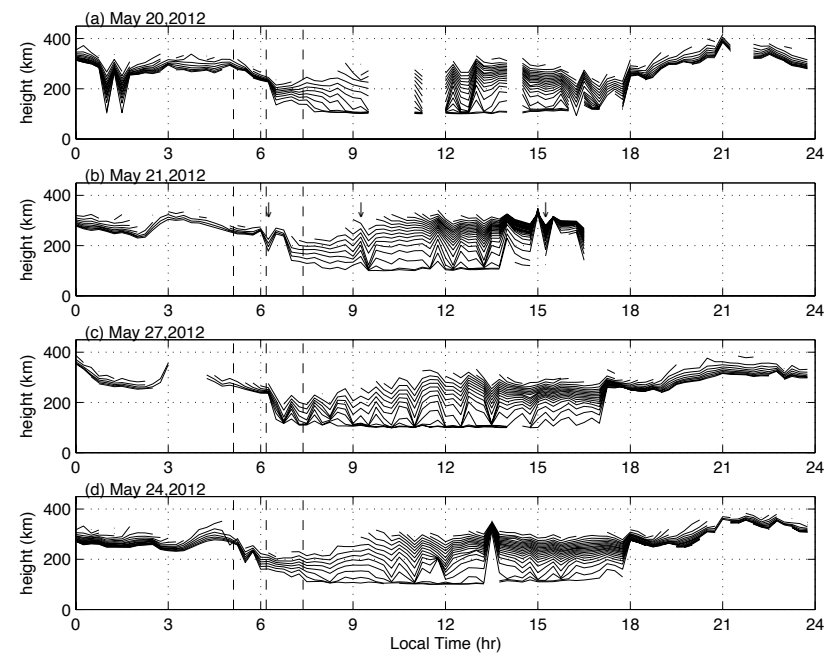

Fig. 7. Diurnal variations in true height at fixed frequencies at Chung-Li ionosonde station on (a) 20, (b) 21, (c) 27, and (d) 24 May 2012. The vertical dashed lines represent the onset, second contact, and ending time of the annular solar eclipse on 21 May 2012.

at all three GPS sites on 21 May, which was most pronounced at the MATZ and TNML stations. The wave-like fluctuation was observed for $3 \mathrm{~h}$ following the end of contact with the annular eclipse on 21 May 2012. For the duration of the event, the geomagnetic field was quiet, and the Dst index values were approximately $-15 \mathrm{nT}$ to $-13 \mathrm{nT}$ between 19 and 22 May 2012. Therefore, the traveling ionospheric disturbance (TID) was not caused by the geomagnetic field disturbances. Compared with the variations on the other days, the result shows no apparent oscillation such as that on 21 May, except for an obvious fluctuation from 17:00 LT to 21:00 LT on 20 May. In other words, the wavelike oscillation from 09:00 LT to 15:00 LT could be associated with the annular eclipse. Conversely, the fluctuation occurring from 17:00 LT to 21:00 LT could be a daily variation caused by the equatorial pre-reversal enhancement and the thermospheric winds. The wave-like oscillation from 09:00 LT to 15:00 LT is similar to the result of Cheng et al. (1992), based on the eclipse occurrence of 23 September 1987. Cheng et al. (1992) showed that the eclipse induced a TID with a propagation velocity assumed to be the speed of sound $\left(300 \mathrm{~m} \mathrm{~s}^{-1}\right)$.

\section{Conclusions}

This study was the first time to observe the response in the $\mathrm{F}$ region of the ionosphere before the formation of the $F_{1}$ layer in an annular solar eclipse over Taiwan. We used the ionospheric parameters $f o \mathrm{~F}_{2}, h m \mathrm{~F}_{2}, H_{m}, B_{0}$, TEC, and $\tau$ to study the effects of the annular solar eclipse during the dawn of 21 May 2012. Our conclusions are as follows: 


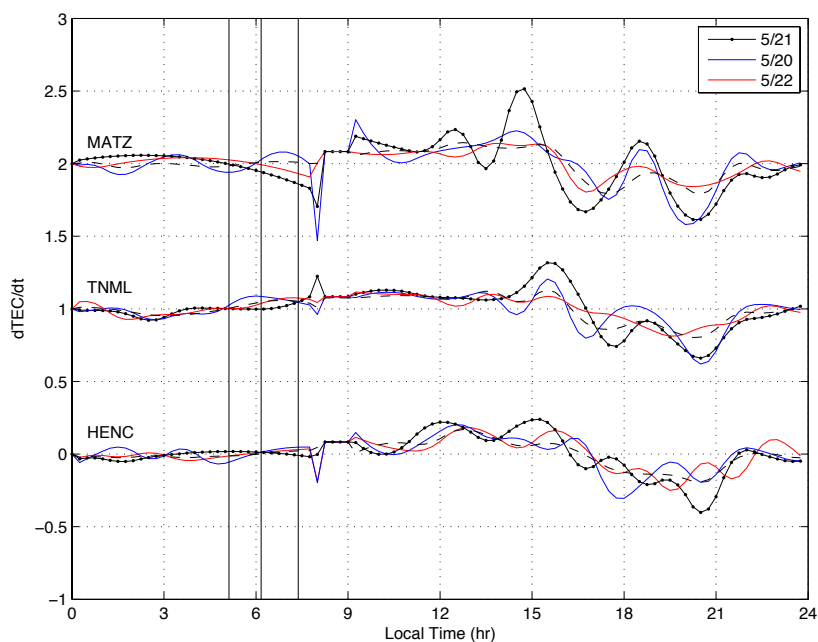

Fig. 8. The TEC rate at three GPS stations (a) MATZ, (b) TNML, and (c) HENC during the solar eclipse on 20 (blue lines), 21 (dotted lines), and 22 (red lines) May 2012, and the monthly mean (dashed lines).

1. The level of the $f o \mathrm{~F}_{2}$ decreased during the period of the eclipse, closely following the fluctuations in local solar radiation. Additionally, the $f o \mathrm{~F}_{2}$ appeared to increase after the eclipse. This contrasts with the study by Cheng et al. (1992) of the ionospheric responses to the annular solar eclipse over Taiwan on 23 September 1987. Evans (1965) suggested two conditions for an increase in the $f o \mathrm{~F}_{2}$ after a solar eclipse. First, the eclipse should be a total or near-total (approximately $>90 \%$ ) eclipse at the $F_{1}$ region. Second, the magnetic dip should be large $(I>60)$. Although neither condition was satisfied in the 21 May 2012 event, similar variations in the $f_{o} \mathrm{~F}_{2}$ were observed over Taiwan $(I=35$, obscuration $=86 \%)$, suggesting that $f o \mathrm{~F}_{2}$ responses are associated with both solar radiation and the equatorial fountain effect.

2. The $h m \mathrm{~F}_{2}, H_{m}$, and $B_{0}$ fluctuated over time during the eclipse period. The variation in height at a fixed frequency propagated from lower to higher altitudes during the eclipse period. After the end of the eclipse, a rapid increase in $H_{m}$ and $B_{0}$ caused variations in the shape of the ionosphere. The topside and bottomside iTEC both increased, but the $h m \mathrm{~F}_{2}$ showed no substantial variation. The increase in iTEC was associated with the solar radiation, which increased the electron density in the $\mathrm{F}$ region. Furthermore, the decreases in $H_{m}$ and $B_{0}$ were attributable to the increases in the $f o \mathrm{~F}_{2}$ and $h m \mathrm{~F}_{2}$ between 13:00 LT and 16:00 LT, which caused the $h m \mathrm{~F}_{2}$ to rise in altitude and decreased the rate of loss.

3. The ionospheric equivalent slab thickness $(\tau)$ decreased during the eclipse period. Jakowski et al. (2008) stated that if the effects of meridional thermospheric winds and electric fields were neglected, the $h m \mathrm{~F}_{2}$ and $\tau$ should increase during the eclipse. However, we observed a substantial decrease in $\tau$. The decrease in $\tau$ is associated with the decrease in electron density and the attenuation of the solar radiation, which prevents diffusion away from the magnetic equator. Additionally, an increase in $B_{0}$ after the eclipse was not observed in $\tau$. $B_{0}$ is associated with solar radiation and the diffusion driven from the equatorial fountain effect, which increases the electron density in the bottomside F region. It is accepted that the shape factor is sensitive to variations in the $\mathrm{H}^{+} / \mathrm{O}^{+}$ ratio at the $F_{2}$ peak height (Davies and Liu, 1991). The $\mathrm{O}^{+}$photoionization rate decreased because the solar obscuration reduced the plasma density in the $\mathrm{F}_{2}$ layer peak height and the topside ionosphere. Therefore, a substantial decrease occurred in $\tau$ but was not observed in $B_{0}$.

4. The TEC decreased substantially along the path of the eclipse (as observed at MATZ and TNML), but no depletion was observed at HENC (outside of the path) during the eclipse period. This shows that local solar radiation is one source of the increasing local ionization loss, because of recombination of the ionosphere. The variation in $\mathrm{dTEC} / \mathrm{d} t$ showed a wave-like oscillation between 12:00 LT and 22:00 LT, and propagation seemed to occur in the path of the eclipse. This was similarly observed in the temporal variation at the fixed frequency on the ground. The wave-like propagation could be triggered by the fast eastward-moving local neutral wind dynamo generated by a lower pressure system and the cold temperatures in the region of maximum obscuration (St. Maurice et al., 2011; Nayak et al., 2012).

5. This study was the first to investigate and compare the variations between the topside and bottomside ionospheric $\mathrm{F}$ region during an annular solar eclipse. We also observed that the $\mathrm{F}_{2}$ layer maximum plasma frequency and $f o \mathrm{~F}_{2}$ decrease simultaneously with solar obscuration. The results show that variation in the $\mathrm{F}_{2}$ layer is dominated by the vertical $\boldsymbol{E} \times \boldsymbol{B}$ and is affected by recombination and production. This explains the behavior of the $f o \mathrm{~F}_{2}, h m \mathrm{~F}_{2}, H_{m}, B_{0}$, and $\tau$ during the 21 May 2012 annular solar eclipse over Taiwan.

Acknowledgements. The authors sincerely thank the Editor and reviewers for their valuable comments and suggestions. We would like to thank the Chung-Li Ionosphere Station National Communications Commission and the Central Weather Bureau of Taiwan for providing the ionograms and GPS data online. This work was supported by National Science Council grants NSC102-2111-M-275001 and NSC102-2119-M-275-001. 
Topical Editor K. Hosokawa thanks T. L. Gulyaeva and one anonymous referee for their help in evaluating this paper.

\section{References}

Adeniyi, J. O., Radicella, S. M., Adimula, I. A., Willoughby, A. A., Oladipo, O. A., and Olawepo, O.: Signature of the 29 March 2006 eclipse on the ionosphere over an equatorial station, J. Geophys. Res., 112, A06314, doi:10.1029/2006JA012197, 2007.

Cheng, K., Huang, Y.-N., and Chen, S.-W.: Ionospheric effects of the solar eclipse of September 23, 1987, around the equatorial anomaly crest region, J. Geophys. Res., 97, 103-111, 1992.

Chimonas, G.: Internal gravity-wave motions induced in the Earth's atmosphere by a solar eclipse, J. Geophys. Res., 75, 5545-5551, doi:10.1029/JA075i028p05545, 1970.

Chimonas, G. and Hines, C. O.: Atmospheric gravity waves induced by a solar eclipse, J. Geophys. Res., 75, 875, doi:10.1029/JA075i004p00875, 1970.

Cohen, E. A.: The study of the effect of solar eclipses on the ionosphere based on satellite beacon observations, Radio Sci., 19, 769-777, 1984.

Davies, K.: Ionospheric Radio, Peter Peregrinus Ltd., London, United Kingdom, 1990.

Davies, K., Liu, X.M.: Ionospheric slab thickness in middle and low-latitudes, Radio Sci., 26, 997-1005, doi:10.1029/91RS00831, 1991.

Evans, J. V.: On the behavior of foF2 during eclipses, J. Geophys. Res., 70, 733-738, 1965.

Gulyaeva, T. L.: Progress in ionospheric informatics based on electron density profile analysis of ionogram, Adv. Sapce Res., 7, 39-48, 1987.

Gulyaeva, T. L.: Variable coupling between the bottomside and topside thickness of the ionosphere, J. Atmos. Sol.-Terr. Phys., 69, 528-536, doi:10.1016/j.jastp.2006.10.015, 2007.

Jakowski, N., Stankov, S. M., Wilken, V., Borries, C., Altadill, D., Chum, J., Buresova, D., Boska, J., Sauli, P., Hruska, F., and Cander, Lj. R.: Ionospheric behavior over Europe during the solar eclipse of 3 October 2005, J. Atmos. Sol.-Terr. Phys., 70, 836853, doi:10.1016/j.jastp.2007.02.016, 2008.
Le, H., Liu, L., Yue, X., and Wan, W.: The ionospheric responses to the 11 August 1999 solar eclipse: observations and modeling, Ann. Geophys., 26, 107-116, doi:10.5194/angeo-26-107-2008, 2008.

Le, H., Liu, L., Yue, X., Wan, W., and Ning, B.: Latitudinal dependence of the ionospheric response to solar eclipse, J. Geosphys. Res., 114, A07308, doi:10.1029/2009JA014072, 2009.

Le, H., Liu, L., Yue, X., and Wan, W.: The ionospheric behavior in conjugate hemispheres during the 3 October 2005 solar eclipse, Ann. Geophys., 27, 179-184, doi:10.5194/angeo-27-179-2009, 2009.

Lee, C.-C. and Reinisch, B. W.: Quiet-condition variations in the scale height at F2-layer peak at Jicamarca during solar minimum and maximum, Ann. Geophys., 25, 2541-2550, doi:10.5194/angeo-25-2541-2007, 2007.

Liu, J. Y., Tsai, H. F., and Jung, T. K.: Total Electron Content Obtained by Using the Global Positioning System, Terr. Atmos. Ocean. Sci., 7, 107-117, 1996.

Liu, J. Y., Sun, Y. Y., Kakinam, Y., Chen, C. H., Lin, C. H., and Tsai, H. F.: Bow and Stern Waves Triggered by the Moon's Shadow Boat, Geophys. Res. Lett., 38, L17109, doi:10.1029/2011GL048805, 2011.

Nayak, C. K., Tiwari, D., Emperumal, K., and Bhattacharyya, A.: The equatorial ionospheric response over Tirunelveli to the 15 January 2010 annular solar eclipse: observations, Ann. Geophys., 30, 1371-1377, doi:10.5194/angeo-30-1371-2012, 2012.

Reinisch, B. W. and Huang, X.: Deducing topside profiles and total electron content from bottomside ionograms, Adv. Space Res., 27, 23-30, 2001.

St.-Maurice, J. P., Ambili, K. M., and Choudhary, R. K.: Local electrodynamics of a solar eclipse at the magnetic equator in the early afternoon hours, Geophys. Res. Lett., 38, L04102, doi:10.1029/2010GL046085, 2011.

Titheridge, J. E.: Ionogram analysis with generalized program POLAN, Rep. UAG-93, World Data Cent. A for Sol.-Terr. Phys., Boulder, Colorado, 1985. 